\title{
Sustainability Capacity of HIV Programs in National Capital Region, Philippines
}

\author{
Euris John B. Cawaling, Dennise U. Cunanan, Racidon P. Bernarte \\ Department of Communication Research, College of Communication, Polytechnic University of the Philippines, \\ Philippines
}

\begin{tabular}{l} 
Article Info \\
\hline Article history: \\
Received Mar 23, 2018 \\
Revised May 1, 2018 \\
Accepted Jun 30, 2018 \\
\hline
\end{tabular}

\section{Keyword:}

Department of Health HIV programs National Capital Region Philippines

Sustainability

\begin{abstract}
Across all the regions in the Philippines, the National Capital Region (NCR) got the highest percentage (37\%) for HIV newly diagnosed cases based from the July-August 2017 HIV/AIDS and ART Registry of the Philippines' report. The National HIV, AIDS, and STI Prevention and Control Program (NASPCP) of the Department of Health (DOH) lead the different health services in the prevention of HIV transmission. Anchored with the NASPCP, different HIV programs of DOH's selected partner institutions and organizations within NCR continuously exist. However, due to the increase on the number of newly diagnosed HIV cases in the country, ensuring its sustainability through assessing the different domains of their program was in need to be studied. This article was based from an undergraduate thesis study which aimed to assess the sustainability capacity of HIV programs among DOH's selected partner institutions and organizations within NCR, Philippines, scoping the eight domains specifically the environmental support, funding stability, partnerships, organizational capacity, program evaluation, program adaptation, communications, and strategic planning. The study used a quantitative approach; 17 government institutions specifically city health offices and 13 non-government HIV-related organizations for a total of 30 respondents. Survey method was utilized using the standardized Program Sustainability Assessment Tool adapted from the Washington University in St. Louis. Based from the results and findings of the study, generally, all the domains were leaning either in great and greater extent as verbally interpreted. From the conclusion, all the domains of the HIV programs of the respondents were sustainable. However, the study recommended to the $\mathrm{DOH}$ and all the HIV program implementers the developed and proposed narrative-type action plan. By that means, future researchers can assess again and see if the NCR achieved the vision of the Health Sector Plan for HIV and STI for the year 2015-2020 to have zero new infections, zero discrimination, and zero AIDS-related deaths.
\end{abstract}

Copyright $@ 2018$ Institute of Advanced Engineering and Science. All rights reserved.

\section{Corresponding Author:}

Racidon P. Bernarte,

Department of Communication Research, College of Communication,

Polytechnic University of the Philippines,

PUP NDC Campus Anonas Street, Sta. Mesa, Manila 1016,

Phone: (Direct Line) 7138412, Philippines.

Email: rpbernarte@pup.edu.ph

\section{INTRODUCTION}

The Philippines, labeled as a "third world country", is also facing the HIV health issue. Based from the article reported by the Reuters, across the Asia-Pacific Region from the past six years, the country had the highest HIV infection growth rate with $140 \%$ increase from the reported cases of new infections [1]. 
In addition, based on the latest Department of Health-Epidemiology Bureau's HIV/AIDS and ART Registry of the Philippines (HARP) report of July-August 2017, thirty-one (31) Filipinos are newly diagnosed with HIV every day; this is far comparable from the figures of last year's twenty-six (26) and the year 2014 with seventeen (17) reported individuals per day. Across all the regions in the Philippines, the National Capital Region (NCR) got $37 \%$ which was the highest percentage for newly diagnosed cases among all the regions [2].

The Philippine government, primarily the Health Department, took this health problem as should be seriously addressed. Through collaborative efforts of the DOH and other health sectors and institutions, they developed the Health Sector Plan for HIV and STI encompassing the years from 2015 to 2020. It was commissioned by the Department of Health-National AIDS/STI Prevention and Control Program (DOHNASPCP) and the World Health Organization (WHO). It was anchored from the findings and recommendations of the 5th AIDS Medium Term Plan: 2011-2016 Philippine Strategic Plan on HIV and AIDS (AMTP) that was developed by the Philippine National AIDS Council (PNAC) [3].

The National HIV, AIDS, and STI Prevention and Control Program (NASPCP) is intended to lead the different health services in the prevention of HIV transmission. The program strategy itself is outlined by different components such as the IEC or the Information, Education and Communication, the STI Diagnosis and Treatments, the Voluntary HIV Counseling and Testing, the Provision of Condoms and Lubricants, the Baseline CD4 Testing for newly-diagnosed HIV Positive Individuals, the Provision of Antiretroviral (ARV) Drugs, and the Monitoring and Evaluation. All these components of the program are anchored to the different specific HIV programs of the government institutions and non-government organizations. If the development of different HIV programs is one of many solutions to prevent the spread of HIV, its sustainability is very important to be prioritized. Addressing the sustainability of the HIV programs is one way to further give efficient HIV preventive services. From the idea of sustainability, it primarily assesses different factors to sustain the program such as the environmental support, the funding stability, the partnerships, the organizational capacity, the program evaluation, the program adaptation, the communications, and the strategic planning. All these were included on the Program Sustainability Assessment Tool of the Center for Public Health System Science-George Warren Brown School of Social Work at Washington University in St. Louis [4-5].

Based on the Health Sector Plan For HIV and STI 2015-2020, the goal by the end of 2020 is to have less than 66 HIV cases per 100,000 populations that will be maintained through the prevention of spreading HIV infection and the provision of treatment care and support that will reduce the impact of the disease on individuals, families, sectors, and communities; the vision is to have zero new infections, zero discrimination, and zero AIDS-related deaths. Assessing and maintaining the sustainability of HIV program/s among government institutions and non-government organizations and developing an action plan to sustain the HIV program are big steps to achieve the country's health sector goal and soon meet its vision [3].

\section{RESEARCH METHOD}

The research study used a quantitative research approach. Through this particular approach, the researchers used to assess larger number of respondents to attain accurate and collective results and findings. The study has also an evaluative purpose; this pertains on the process of evaluating and assessing the sustainability of the HIV Programs among different institutions and organizations. The study used the form of survey as the type of research method which employed to answer the research question and objectives.

\subsection{Population, Sample Size, and Sampling Technique}

There were two target populations for this research study. One was the population for the partner institutions of the Department of Health which pertains to government institutions; specifically the City Health Offices or Social Hygiene Clinics across the National Capital Region. The other one was the partner organizations of the Department of Health which pertains to non-government organizations or support groups that have HIV-related advocacy. There were 17 total partner government institutions of the Department of Health that have their own specific HIV program/s which are all anchored to the National HIV/STI Prevention Program of the country's health department. Based on the record of the Philippine Statistics Authority, the National Capital Region has sixteen (16) cities and one (1) municipality. [6] On the other hand, there was no definite number for the population of those partner non-government organizations of the DOH with HIV-related advocacy. However, the Philippine National AIDS Council suggested some of those organizations and was approved by the DOH-National AIDS/STI Prevention and Control Program (NASPCP); a total of thirteen (13) selected partner NGOs or support groups. The research study used a non-probability sampling technique, specifically the purposive sampling technique to purposely sought particular target respondents. 


\subsection{Description of Respondents}

Primarily, the respondents of this research study were the Department of Health's selected partner institutions and organizations. City health offices particularly social hygiene clinics across National Capital Region are the target government institutions; medical coordinator/medical officer/physician was the target respondent which mainly has the highest knowledge in terms of the HIV program. As for the nongovernment organization or those HIV-related advocate groups, the target respondent was their program manager or if not available, any program coordinator that also has the highest knowledge in HIV program. Table 1 shows the names of the respondents, their represented institution/ organization and their position.

Table 1. Description of the Respondents

\begin{tabular}{|c|c|c|}
\hline Institution/ Organization & Name of the respondent & Position \\
\hline Action for Health Initiatives (ACHIEVE) & Junelyn R. Tabelin & Project Coordinator \\
\hline AIDS Society of the Philippines & Jesus A. Ramirez & Program manager \\
\hline City Health Office of Caloocan & Nerissa P. Bello & Med. Tech. III \\
\hline City Health Office of Makati & Teresita P. Pagcaliwagan & HIV Nurse Coordinator \\
\hline City Health Office of Malabon & Cecilia M. Gozon, MD & HIV/AIDS Medical Coordinator \\
\hline City Health Office of Mandaluyong & Yolanda D. Tuaǹo, MD & Medical Consultant \\
\hline City Health Office of Manila & Diana Mendoza, MD & Medical Officer V \\
\hline City Health Office of Marikina & Honnielyn Fernando & Acct. CHO/ STI/HIV/AIDS Prorgam Coordinator \\
\hline City Health Office of Muntinlupa & Aurora Ferrer, MD & STI/HIV/AIDS Medical Coordinator \\
\hline City Health Office of Navotas & Henry Tiǹo, MD & STI/HIV Rural Medical Officer \\
\hline City Health Office of Las Pinas & Dr. Maria Alinea D. Salatan & Physician-in-charge \\
\hline City Health Office of Quezon City & John Jardenil & Secretary \\
\hline City Health Office of Paranaque & (Confidential) & Social Hygiene Clinic Nurse \\
\hline City Health Office of Pasay & Joan Carlota Ranieses, MD & Medical Officer V \\
\hline City Health Office of Pasig & Roque, MD & HIV Coordinator \\
\hline City Health Office of San Juan & Celine Adante & Social Hygiene Clinic Nurse \\
\hline City Health Office of Taguig & Diwata Arriola, MD & Medical Coordinator \\
\hline City Health Office of Valenzuela & Josephine G. Diaz, MD & STI/HIV/AIDS Medical Coordinator \\
\hline $\begin{array}{l}\text { Family Planning Organization of the Philippines } \\
\text { (FPOP) }\end{array}$ & Nandy Senoc & Executive Director \\
\hline HIV/ AIDS Support House Inc. (HASH) & Desi Andrew Ching & Executive Director \\
\hline Love yourself Inc. & Josif Cade & Program Manager \\
\hline Municipality of Pateros & Imelda P.C. & Medical Officer \\
\hline $\begin{array}{l}\text { Philippine NGO Council on Population Health } \\
\text { and Welfare Inc (PNGOC) }\end{array}$ & Ralph Ivan J. Samson, RN & Training Officer \\
\hline Pilipinas Shell Foundation & Julius Elopre & $\begin{array}{l}\text { Monitoring and Evaluation Officer for Philippine } \\
\text { Business Sector Response to HIV and AIDS } \\
\text { Programs }\end{array}$ \\
\hline Pinoy Plus Association Inc. & Noel Quinto & Former President/ Project Coordinator \\
\hline Positibong Marino Philippines Inc. & Vilton Jude Adao & Secretary \\
\hline $\begin{array}{l}\text { Positive Action Foundation of the Philippines } \\
\text { (PAFPI) }\end{array}$ & Rodel Navarra & Chief Operation Officer \\
\hline $\begin{array}{l}\text { Sustainaed Health Initiatives of the Philippines } \\
\text { (SHIP) }\end{array}$ & (Confidential) & (Confidential) \\
\hline $\begin{array}{l}\text { The Project Red Ribbon Care Management } \\
\text { Foundation Inc. }\end{array}$ & Rogeselle B. Monton & Administrator \\
\hline TLF Share Collectives Inc & Marcy Oculto & Program Officer \\
\hline
\end{tabular}

\subsection{Research Instrument}

The research study used the Program Sustainability Assessment Tool (PSAT) as the suitable research instrument which was adapted from the Center for Public Health Systems Science-George Warren Brown School of Social Work at the Washington University in Saint Louis [5].

\subsection{Data-Gathering Procedure}

This research study collects the data by personally distributing the modified HIV Program Sustainability Assessment Tool as survey questionnaires to the target respondents. The initial step is to send an email or to give a letter of intent to a specific addressee; for some instances, they send back their response together with the attached answered survey form through online.

\subsection{Research Instrument}

As for the statistical treatment of the gathered data, the researchers encoded all the information and primary data on the online website of the Program Sustainability Assessment Tool. The online website of this particular tool will assess the program's capacity for sustainability; it will also provide figures and automated 
summary report of the overall sustainability. However, to further produce statistically accurate result and findings, all the data are run through Statistical Package for the Social Sciences (SPSS) software.

\section{RESULTS AND ANALYSIS}

Generally, all the findings of the eight domains measured through the Program Sustainability Assessment Tool were leaning towards positive side. The first four domains such as the environmental support, financial stability, partnerships, and organizational capacity were verbally interpreted as having "great extent" while the remaining four such as the program evaluation, program adaptation, communications, and strategic planning were verbally interpreted as having "greater extent". Table 2 shows summary of sustainability capacity of all domains.

Table 2. Summary of Sustainability Capacity of all Domains

\begin{tabular}{lccc}
\hline \multicolumn{1}{c}{ Statements } & Gen. Weighted Mean & Gen. Weighted SD & Verbal Interpretation \\
\hline Environmental Support. & 5.14 & 1.32 & Great extent \\
Funding Stability & 5.07 & 1.33 & Great extent \\
Partnerships & 5.22 & 1.39 & Great extent \\
Organizational Capacity & 5.30 & 1.57 & Great extent \\
Program Evaluation & 5.63 & .94 & Greater extent \\
Program Adaptation & 5.68 & 1.16 & Greater extent \\
Communications & 5.74 & 1.20 & Greater extent \\
Strategic Planning & 5.49 & & Greater extent \\
\hline $1.00-1.86$ (No extent) & & & \\
$1.87-2.73$ (Lesser extent) & & & \\
$2.74-3.60$ (Less extent) & & & \\
$3.61-4.47$ (Some extent) & & & \\
$4.48-5.34$ (Great extent) & & & \\
$5.35-6.21$ (Greater extent) & & &
\end{tabular}

\subsection{Environmental Support}

The data implies that when it comes to sustainability capacity as to environmental support, four out of five statements had great extent when it comes to the likelihood scale. On the other hand, greater extent was shown in the idea that the HIV programs have leadership support from outside of the organization. In that sense, leaders such as political officials, businessmen and even champions that are outside the institution or organization were strongly supportive when it comes to HIV advocacy.

From the findings of this particular domain (environmental support), it clearly supports the 5th AIDS Medium Term Plan (AMTP): 2011-2016 Philippine Strategic Plan on HIV and AIDS regarding on the idea of both national and local response. The 5th AMTP stated different government institutions and non-government organizations which primarily initiated by the Philippine National AIDS Council (PNAC). The PNAC is labeled as the "driver of the integrated and comprehensive country response" to address and fight the HIV and AIDS. Together with PNAC, the following are the national line agencies which continuously working to combat the HIV/AIDS: (1) the Department of Health (DOH) as the lead agency in our country that provides direct health services and surveillance, under the DOH are the HIV and AIDS Core Teams (HACT) which specifically assigned in different hospitals to immediately respond on the needs for the implementation of the program, the Epidemiology Bureau (EB) which serves as the surveillance system which collects the data regarding on the HIV/AIDS reported cases, and also the National AIDS, STI Prevention and Control Program (NASPCP) as a working group that manages the health sector program in HIV, AIDS and STI; (2) the Department of Labor and Employment (DOLE) is responsible to all the labor sectors in implementing the policies regarding in the HIV prevention; (3) the Department of Education (DepEd) and the Commission on Higher Education (CHED) are both information-giving agencies which mainly deals the three levels of education (primary, secondary, tertiary), it ensures to educate the students about the HIV/ AIDS issue; (4) the Department of Social Welfare and Development (DSWD) provides relevant welfare services and social needs of those people living with HIV; (5) the Department of Foreign Affairs (DFA) also provides services among Overseas Filipino Workers (OFWs) with HIV and also take part in discussing HIV/AIDS issues with other international agencies through different fora; (6) the Department of Interior and Local Government (DILG) leads the implementation of the policies at the local level which addresses the HIV/AIDS problem; (7) the National Economic and Development Authority (NEDA) is responsible in the National AIDS Spending Assessment (NASA), the agency also monitors and evaluates HIV/AIDS-related reports; (8) the Local Government Units (LGUs) primary implements and apply all the 
HIV/AIDS-related policies at the local level; (9) the Civil Society Organizations (CSOs) help the national agencies and LGUs to implement the HIV program through community-based approach; and lastly (10) the Faith-Based Organizations (FBOs) such as the National Secretariat for Social Action and Justice of the Catholic Bishop Conference of the Philippines (CBCP) initiates different HIV-related preventive activities; these agencies together with other HIV-related advocates either an individual, a government institution or a non-government organization could support the HIV programs [7].

Several local and international organizations with HIV-related advocacy are continuously partnering with the LGUs and other NGOs with the same advocacy. One of them is the Global Fund (GF), it is a multibillion international financing mechanism which primarily operates as a partnership with the government, civil society, private sectors, and affected communities, the GF directly gives money to finance different activities and interventions related in preventing different diseases such as the HIV/AIDS (The Global Fund To Fight AIDS, TB \& Malaria Philippine country Coordinating Mechanism Briefer, n.d.). In partnership with the Global Fund, another international organization is the Save the Children, it is entitled as the 'world's leading independent children's organization'; for over 30 years in the country, one of many aspects of their work is for the children to access quality health services [8].

Other environmental support for the HIV program were mentioned as potential resources in the local government of Quezon City, these are the United States Agency International Development (USAID), Japan International Cooperation Agency (JICA), United Nations (UN), World Bank, Asian Development Bank, and International HIV/AIDS Alliance [9].

\subsection{Funding Stability}

Generally, the data implies that the respondents had great extent in terms of their funding. In line with this, the Philippine government proportionally manages the allocation of funds and budget regarding on the HIV programs.

In support to the idea above, the 2017 People's Proposed Budget of the Department of Budget and Management allotted P146.18 billion pesos for the health sector. This includes the P24.6 billion pesos apportioned to have preventive and promotive health; the P1.6 billion pesos part is specifically allotted to diagnosed and treat different infectious diseases such as the HIV/AIDS [10].

\subsection{Partnerships}

The data implies that in terms of the partnership within the HIV programs, generally, the DOH's partner institutions and organizations had their own capability to gather connections and resources among other community members. The figures were leaning in the positive side wherein it indicates that DOH's partner institutions and organizations together with other HIV-related advocates or groups sustained their partnership aspect.

Furthermore, the results and findings of this study were reflected from two guiding principles in the Strategic Plan for 2011 - 2016 of the 5th AIDS Medium Term Plan (2011-2016):

Community Participation: Invoke the rights of citizens (communities, civil society organizations, and networks of people living with HIV) to actively participate and engage the state in addressing their needs and concerns [7].

Partnerships: Facilitate comprehensive stakeholder consultations and dialogue, encouraging partnership-driven development in the planning and implementation of relevant HIV and AIDS responses involving all spheres [7].

\subsection{Organizational Capacity}

The data reflects how the DOH's partner organizations and institutions with HIV programs were capable enough as based from the result in terms of their functional ability. These show that the selected respondents were able to effectively manage the HIV program.

The number of personnel or staff within a particular institution or organization is aligned on the "Management Structure of Health Sector" when it comes to delivering the services of the HIV program. In the national level, there are different positions such as the program manager and staffs of the National AIDS/STI Prevention and Control Program (NASPCP), Epidemiology Bureau (EB), and the STS/AIDS Central Cooperative Laboratory (SACCL); in the sub-national level, there are regional officers, regional coordinators, peer educators, and site implementation officers for Center for Health Development Regional level; lastly in the local level are the medical coordinators/medical officers/physicians, nurses, med. tech., support staffs, peer educators, and site implementation officers within Social Hygiene Clinics [3]. 


\subsection{Program Evaluation}

Generally, the respondents were continuously monitoring and evaluating different aspects of the HIV program to ensure its effectiveness and capability to be sustained. In line with the idea of program evaluation, the Health Sector Plan for HIV and STI 2015-2020 included a Reporting Flow that emphasizes different program indicators which are responsible to monitor and evaluate the HIV program and also provide data to be submitted by PNAC to the global community [3]. Moreover, the 5th AIDS Medium Term Plan (AMTP): 2011-2016 Philippine Strategic Plan on HIV and AIDS included an illustration of the HIV and AIDS Monitoring and Evaluation Flow; this highlights the process of data reporting from municipal, provincial to national levels [7].

\subsection{Program Adaptation}

The data implies that when it comes to program adaptation, the DOH's partner institutions and organizations were able to embrace the changes and adapt suitable and applicable innovations. The average score of 6 interpreted as greater extent with all the statements included from the domain pertaining to program adaptation. There are so many things that the DOH's partner institutions and organizations could adapt, with the basis of recent researches and evidences, to achieve the effectiveness of the HIV program. The government considered the recommendations of the World Health Organization regarding on the provision of "Essential and Comprehensive Package of Services" for Key Populations (sex workers, men who have sex with men, people who inject drugs, youth). The Essential Package of Services encompasses human skills, drugs, equipment, and other resources that will help to conduct the interventions; one example is the condom and lubricant promotion and provision [3].

\subsection{Communications}

Generally, the data implies that strategic communications had greater extent as assessed by the respondents. Through different means of communication, the HIV programs of different DOH's partner institutions and organizations were positively broadcast to the public. In that sense, these institutions and organizations were able to continue the increase of awareness towards the HIV issue.

In terms of communications, the HIV programs of the DOH's partner institutions and organizations were anchored from the Strategy 2 of the Health Sector Plan For HIV and STI 2015-2020 which pertains in "Health Promotion and Communication on HIV and STI Prevention and Care Services". The idea of "peer outreach' was suggested as an intervention to increase the coverage of the people, this by the means of giving information, educating and communicating [3].

\subsection{Startegic Planning}

The data implies that, in general, those DOH's partner institutions and organizations were able to develop strategic planning. This reflects how all the elements from the sustainability assessment were leaning towards the positive side, it means that the effectiveness of the HIV program falls either in great or greater extent of sustainability. Based on the data interpretation, it only manifested that DOH's partner institutions and organizations' strategic planning towards HIV programs were anchored from the Health Sector Plan For HIV and STI 2015-2020; it includes four specific strategies that addresses the HIV issue in the country; these are (1) Continuum of HIV/STI prevention, diagnosis, treatment and care service to Key Populations, (2) Health promotion and communication on HIV and STI prevention and care services, (3) Enhance strategic information systems, and (4) Strengthened health system platform for broader health outcomes [3].

\subsection{Over-all Discussion of Results}

When it comes to the domain of environmental support, the findings of this study somehow debunked the idea mentioned from the Health Sector Plan for HIV and STI 2015-2017. It was stated under the HIV \& STI Situation: Country's Response that the HIV control and prevention interventions had decentralized health systems, it was said to be "critical to involve the Local Government Units (LGUs) which were the primary implementers of the health programs". On the other hand, it was also stated from the above health sector plan that due to the existence of HIV and STI prevention social hygiene clinics which were counted 119 in total; there was increase awareness among local political leaders regarding the HIV health issue. In that sense, the environmental support aspect of the HIV program will continue be sustained if the political/ local leaders greatly support the HIV program through their awareness. Furthermore, from the news article of The Philippine Star, the House of Representatives committee on health approved the "Philippine HIV and AIDS Policy Act" or a "bill that seeks to strengthen the fight against human immunodeficiency virus (HIV) and acquired immune deficiency syndrome (AIDS) amid rising new cases in the country". [11] This only shows that even the government in national level importantly addresses the HIV issue. 
There was a consistent positive view in dealing with the funding stability between the result of this study and the current situation in the country. The study results in general were verbally interpreted as having "great extent" when it comes to funding stability of HIV programs. This finding was supported by a news article entitled "Philippines' HIV funding request to the Global Fund contains a good mix of prevention and treatment: TRP"; there was an increased in Domestic Budget Allocations for HIV from \$9.6 million in 2015 to $\$ 46.0$ million in 2017 , the said "co-financing commitments" which explained as the direct government contributions to the HIV program represented an additional \$188.3 million for the years 2018-2020. Though there are a lot of funding resources both local and international, there should always be a regular evaluation and assessment to see if the funds are appropriately given for the HIV programs and directly benefited by the public [12].

Through the data gathering period, researchers had several opportunities to talk to some people who work in social hygiene clinics for the HIV program. Even these conversations were not recorded and not officially part of the research methodology, many insights were brought up as these people narrated some stories. These community people are the primary partners for the HIV programs; they were somehow labeled as volunteers in a way that they give extra time and effort to conduct different activities inline on the HIV program, some of them get allowances from the city government. The idea of partnership in this research study is not just about those groups outside the community who are supporting and partnering for the HIV program but also those individuals who continuously exerting efforts to help implement the HIV program in their community.

In terms of organizational capacity, several city health offices and non-government organizations which are included in this study had functional workers; from highest to lowest positions, HIV program implementers were doing their respective tasks to accomplish the goal/s of their institution or organization regarding the HIV program. Generally, the program evaluation and program adaptation were both actively practicing by those selected institutions and organizations of this research study. The evidence of this claim is the reports of the institution/organization submitted to the Department of Health and the feedbacks of the health department to those institutions/organizations.

When it comes to communication strategies, HIV program implementers continue to be engaged to all the members of the community in disseminating the awareness and other HV-related information. On the other hand, some non-government organizations had opportunities to use the social media and other media platforms to communicate to their target audience. Lastly, the results and findings of this research study regarding the strategic planning of the respondents only manifested that those selected institutions and organizations were developing strategized plan to ensure the effectiveness of their specific HIV program.

\section{CONCLUSION}

Provide a statement that what is expected, as stated in the "Introduction" chapter can ultimately result in "Results and Discussion" chapter, so there is compatibility. Moreover, it can also be added the prospect of the development of research results and application prospects of further studies into the next (based on result and discussion).

With the summarized sets of findings acquired from the data gathered among the Department of Health's selected partner institutions and organizations, the researchers have drawn the following study conclusions. Based on the Department of Health's selected partner institutions and organizations' assessment on the sustainability capacity of their HIV program with two general remark of "great extent" and "greater extent", the researchers concluded that the respondents' respective HIV programs were sustained when it comes to various domains. However, to have "zero new infections, zero discrimination, zero AIDS-related deaths"; the researchers' presumption that this could be achieved if the HIV programs are all in the greater extent or full extent in the long term process.

In addition, it was also concluded that the increase of awareness of the political leaders and other champions of the HIV advocacy may strengthen the environmental support of a specific HIV program in a community. The researchers also established a conclusion regarding the financial stability of the institutions and organizations, though the findings reflected a financially stable HIV programs, institutions and organizations were still needed more budget to allocate more in the activities and other interventions included in the HIV program. As the track of those newly diagnosed HIV reported cases increase every year, partnerships from the local community and more workers for the organizational capability of the HIV program should have. Furthermore, the researchers also concluded that the program evaluation, program adaptation, communications, and strategic planning of the HIV program should continue to strengthen and practice by the program implementers, this will helps to maintain the positive assessment of the latter domains. The researchers concluded that the proposed HIV Action Plan will help the program implementers 
and other government agencies such as the Department of Health and the Philippine National AIDS Council to contribute another data and understanding when it comes to sustainability of the HIV programs.

\section{ACKNOWLEDGEMENTS}

The authors would like to express their deepest gratitude to all the individuals or any group who supported their research study. Mainly, the Department of Health - National AIDS/STI Prevention and Control Program (NASPCP) and all the respondents from city health offices and non-government organizations who participated in the conduct of suvey.

\section{REFERENCES}

[1] Philippines has highest HIV infection growth rate in Asia-Pacific: U.N., 2017, August 01, Reuters. Retrieved from https://goo.g1/Ux85LG

[2] HIV/AIDS and ART Registry of the Philippines, 2017, July-August, National HIV/AIDS \& STI Surveillance and Strategic Information Unit. Epidemiology Bureau. Department of Health.

[3] Health Sector Plan For HIV and STI 2015-2020. (n.d.). National HIV, AIDS and STI Prevention and Control Program. Disease Prevention and Control Bureau. Department of Health.

[4] Department of Health. (n.d.). HIV/STI Prevention Program. Retrieved from https://goo.gl/uBt9dE

[5] Luke, D., Calhoun, A., Robichaux, C.,Elliott, M. \& Moreland-Russel, S. 2014, "The Program Sustainability Assessment Tool: A New Instrument for Public Health Programs", Prev. Chronic. Dis.DOI: 10.5888/pcd11.130184

[6] Philippine Statistics Authority. (2016). Population of the National Capital Region (Based on the 2015 Census of Population). Retrieved from https://psa.gov.ph/content/population-national-capital-region-based-2015-censuspopulation-0

[7] 5th AIDS Medium Term Plan: 2011-2016 Philippine Strategic Plan on HIV and AIDS (n.d.). Philippine National AIDS Council.

[8] Save the Children, 2017, HIV and AIDS Programs. Retrieved from https://goo.gl/ma3iXs

[9] Wellesly, S. (n.d.). The Development and Utilization of a Local AIDS Investment Plan: The Experience of Quezon City Health. Retrieved from https://goo.gl/jzbozN

[10] 2017 People's Proposed Budget. (n.d.) Department of Budget and Management. Retrieved from https://goo.gl/iWpX4R

[11] Crisostomo, S. and Diaz, J., 6 August 2017, House bill for stronger HIV/AIDS fight approved. The Philippine Star. Retrieved from https://goo.gl/6Wcf3J

[12] Mienies, K., 7 November 2017, Philippines' HIV funding request to the Global Fund contains a good mix of prevention and treatment: TRP. Independent Observer of the Global Fund. Retrieved from https://goo.gl/wDKxxs. 